\title{
On the Existence of Poncelet-Morley Points in Euclidean Geometry
}

\author{
${ }^{1}$ Joseph Dongho ${ }^{1}$, Nguimbous F. L. ${ }^{1} \&$ Teyou M. R. ${ }^{1}$ \\ ${ }^{1}$ Department of Mathematics and Computer Science, University of Maroua \\ Correspondence: Joseph Dongho, Department of Mathematics and Computer Sciences, Faculty of Science, University of \\ Maroua, Po-Box 163 Maroua-Domayo, Cameroon. E-mail: josephdongho@yahoo.fr
}

\author{
Received: December 16, 2016 Accepted: March 30, 2017 Online Published: May 25, 2017 \\ doi:10.5539/jmr.v9n3p68 ～URL: https://doi.org/10.5539/jmr.v9n3p68
}

\begin{abstract}
In this paper, we prove the existence of the Poncelet-Morley point for a given elliptic configuration. The paper ends with an application of such a point in angle trisection problem.
\end{abstract}

Keywords: Poncelet theorem, Morley Theorem, Poncelet-Morpey point, trisection, Galois theory, congruent figure

\section{Msc200: 51M04, 51M05, 11R32, 12F10 and 97G99}

\section{Introduction}

Trisection is a classic problem of compass and straightedge constructions of ancient Greek mathematics. It concerns the construction of an angle equal to one third of a given arbitrary angle, using only two tools: an unmarked straightedge, and a compass. The problem as stated is generally impossible to solve, as shown by Pierre Wantzel in (Arnaudiès J. M. \& Fraysse H., 1990). Note that the fact that there is no way to trisect an angle in general with just a compass and a straightedge does not mean that there is no trisecting angle: for example, it is relatively straightforward to trisect a right angle, that is, to construct an angle of measure 30 degrees. It is, however, possible to trisect an arbitrary angle by using tools other than straightedge and compass. We can also prove using trigonometry and algebraic tools that it is possible to trisect an angle; see solution of Morley problem in (Jean Fresnel, 1996). In plane geometry, Morley's trisector theorem states that in any triangle, the three points of intersection of the adjacent angle trisector form an equilateral triangle, called the first Morley triangle or simply the Morley triangle. The theorem was discovered in 1899 by Frank Morley. It has various generalizations; in particular, if all of the trisectors are intersected, one obtains four other equilateral triangles. A simple proof of the problem is given in (Jean Fresn, 1996) but it remains difficult to define a construction programme associated to an arbitrary triangle.

Our aim is to propose a new geometrical tool and use it to prove that there are angles which cannot be trisected. Of course, we also need the Poncelet result to prove the existence of our main tool called the Poncelet-Morley point for a given configuration.

We recall that it was in 1813 during his captivity as war prisoner that J. V. Poncelet discovered that if $C_{1}$ and $C_{2}$ are non degenerate conics in general position which neither meet nor intersect, if there is an n-sided polygon inscribed in $C_{1}$ and circumscribed about $C_{2}$, then for any point $P$ of $C_{1}$ there exist an $n$-sided polygon also inscribed in $C_{1}$ and circumscribed about $C_{2}$ which has $P$ as one of its vertices. We can then define our main tool. Let $\left(\mathcal{E}_{l}\right)$ be a non degenerate ellipse with focus $F$ and $F^{\prime}, X$ an external point of $\left(\mathcal{E}_{l}\right), T_{1}$ and $T_{2}$ tangents on $\left(\mathcal{E}_{l}\right)$ respectively at $M_{1}$ and $M_{2} . X$ is called a Poncelet-Morley point if $\widehat{M_{1} X F^{\prime}} \cong \widehat{M_{1} X F^{\prime}} \cong \widehat{F^{\prime} X F} \cong \widehat{F X M_{2}}$. We prove the existence of a Poncelet-Morley point for a given configuration. We also prove that for any elliptic configuration, there are two symmetric Poncelet-Morley points. We use the Poncelet-Morley point to prove that it is not possible to trisect all angles.

\footnotetext{
${ }^{1}$ J. D Was partially supported by IHES and MINESUP
} 


\section{Morley Point Associated to an Ellipse Configuration}

Let $\left(\mathcal{E}_{l}\right)$ be a non degenerate ellipse with focus $F$ and $F^{\prime}$ and let $X$ be an external point of $\left(\mathcal{E}_{l}\right)$. $X$ is called a Morley point associated to $\left(\mathcal{E}_{l}\right)$ if there exist two points $M$ and $N$ on $\left(\mathcal{E}_{l}\right)$ such that $\widehat{M X F^{\prime}}=\widehat{F^{\prime} X F}=\widehat{F X N}$.

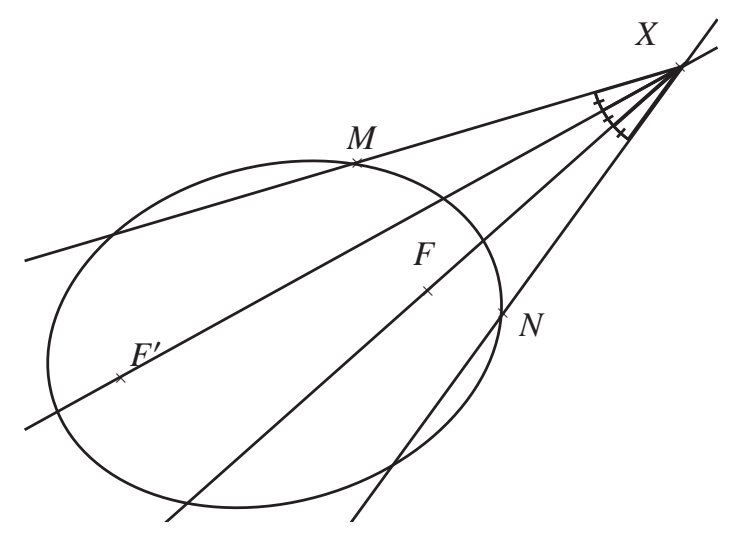

Proposition 1. Each non degenerate ellipse $\left(\mathcal{E}_{l}\right)$ has Morley points.

Proof. If $\left(\mathcal{E}_{l}\right)$ is a non degenerate ellipse of focus $F$ and $F^{\prime}$ then $F F^{\prime} \neq 0$. Therefore the circle $(C)$ with center $F^{\prime}$ and radius $F F^{\prime}$ is non degenerate and it intersects $\left(\mathcal{E}_{l}\right)$. Let $M \in C \cap \mathcal{E}_{l}$; then $M F^{\prime}=F F^{\prime}$ and $\overrightarrow{F^{\prime} M} ; \overrightarrow{F^{\prime} F} \neq 0$. Let $(\Delta)$ be the bisector of $\overrightarrow{F^{\prime} M} ; \overrightarrow{F^{\prime} F}$ and let $X \in(\Delta)$ such that $X$ is not in the domain bounded by $\mathcal{E}_{l}$. The triangle $\Delta_{F F^{\prime} M}$ is isosceles at $F^{\prime}$. Therefore, $(\Delta)$ is the mediator of segment $[F M]$. Moreover, $X \in(\Delta)$ and $\Delta_{X F M}$ is an isosceles triangle in $X$. Then $\overrightarrow{X M} ; \overrightarrow{X F^{\prime}}=\overrightarrow{X F^{\prime} ; \overrightarrow{X F}}$. Let $\left(\Delta^{\prime}\right)$ be the symmetric of $(\Delta)$ with respect to $(F X)$. If $A$ is a point of $\left(\Delta^{\prime}\right)$ then $\overrightarrow{X M} ; \overrightarrow{X F^{\prime}}=\overrightarrow{X F^{\prime} ; \overrightarrow{X F}}=\overrightarrow{X F} ; \overrightarrow{X A}$ and $\Delta^{\prime} \cap \mathcal{E}_{l} \neq \emptyset$. Indeed, if $\left(T_{1}\right)$ and $\left(T_{2}\right)$ are tangents to $\left(\mathcal{E}_{l}\right)$ respectively at $M_{1}$ and $M_{2}$ passing through $X$ and $(X M) \cap\left(\mathcal{E}_{l}\right) \neq \emptyset$, then $(X M)$ is in interior region bounded by $\left(T_{1}\right)$ and $\left(T_{2}\right)$. We shall prove that $\left(\Delta^{\prime}\right)$ is interior with $\left(T_{1}\right)$ and $\left(T_{2}\right)$. Since $M_{1}=T_{1} \cap \mathcal{E}_{l}$ and $M_{2}=T_{2} \cap \mathcal{E}_{l}$ then, according to the second Poncelet theorem, we have $\overrightarrow{X F} ; \overrightarrow{X M_{2}}=\overrightarrow{X M_{1}} ; \overrightarrow{X F^{\prime}}$. Therefore, $\overrightarrow{X F} ; \overrightarrow{X A}+\overrightarrow{X A} ; \overrightarrow{X M_{2}}=\overrightarrow{X M_{1} ; \overline{X M}}+\overrightarrow{X M} ; \overrightarrow{X F^{\prime}}$ and then $\overrightarrow{X A} ; \overrightarrow{X M_{2}}=\overrightarrow{X M_{1}} ; \overrightarrow{X M}$. If $(\Gamma)$ denotes the bisector of $\overrightarrow{X M_{1}} ; \overrightarrow{X M_{2}}$, then $\left(X M_{1}\right)$ and $\left(X M_{2}\right)$ are symmetric relatively to $(\Gamma)$. If $\vec{u}$ is a direction of $(\Gamma)$, then $\overrightarrow{X M_{1}} ; \vec{u}=\vec{u} ; \overrightarrow{X M_{2}}$ and then $\overrightarrow{X A} ; \overrightarrow{X M_{2}}=\overrightarrow{X M_{1}} ; \overrightarrow{X M}$ i.e; $\overrightarrow{\overrightarrow{X A} ; \vec{u}}+\overrightarrow{\vec{u} ; \overrightarrow{X M_{2}}}=\overrightarrow{X M_{1}} ; \vec{u}+\overrightarrow{\vec{u}} ; \overrightarrow{X M}$ i.e; $\overrightarrow{\overrightarrow{X A}} ; \vec{u}=\overrightarrow{\vec{u}} ; \overrightarrow{X M}$

Therefore $(\Gamma)$ is a bisector of $\widehat{X A} ; \overrightarrow{X M}$ i.e; $(X A)$ and $(X M)$ are symmetric relatively to $\Gamma$.

Since $(X M)$ is in the interior region bounded by $\left(T_{1}\right)$ and $\left(T_{2}\right)$ and $(X A)$ is the symmetric of $X M$ relatively to $(\Gamma)$ then $(X A)=\left(\Delta^{\prime}\right)$ is in the interior bounded by $\left(T_{1}\right)$ and $\left(T_{2}\right)$. Therefore $\left(\Delta^{\prime}\right) \cap\left(\mathcal{E}_{l}\right) \neq \emptyset$.

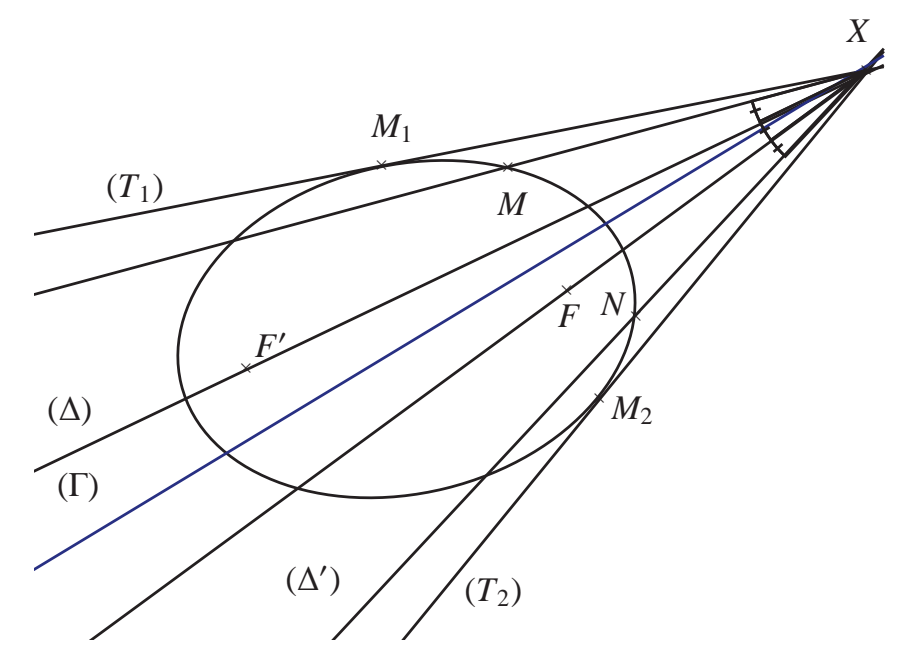


Taking $N\left(\Delta^{\prime}\right) \cap\left(\mathcal{E}_{l}\right)$ we have $\widehat{X \overrightarrow{X N} ; \overrightarrow{X F}}=\widehat{X F} ; \overrightarrow{X F^{\prime}}=\widehat{X F^{\prime} ; \overrightarrow{X M}}$. Therefore $X$ is a Morley point associated to $\left(\mathcal{E}_{l}\right)$.

\section{Poncelet-Morley Points}

Let $\left(\mathcal{E}_{l}\right)$ be an ellipse with focus $F$ and $F^{\prime}, X$ an external point of $\left(\mathcal{E}_{l}\right), T_{1}$ and $T_{2}$ tangent to $\left(\mathcal{E}_{l}\right)$ respectively at $M_{1}$ and $M_{2} . X$ is called Poncelet-Morley point if $\widehat{M_{1} X F^{\prime}} \cong \widehat{F^{\prime} X F^{\prime}} \cong \widehat{F X M_{2}}$.

Let us note that a Poncelet-Morley point of the ellipse $\mathcal{E}_{l}$ is the intersection of two tangents to $\mathcal{E}_{l}$. Given any tangent $T_{1}$ to an ellipse $\mathcal{E}_{l}$, determining a Poncelet-Morley point associated to a giving configuration is equivalent to construct another tangent $T_{2}$ to $\mathcal{E}_{l}$ such that $X=T_{1} \cap T_{2}$ is a Morley point associated to $\mathcal{E}_{l}$.

Proposition 2. Let $\left(\mathcal{E}_{l}\right)$ be an ellipse with focus $F$ and $F^{\prime} ; M$ a point of $\left(\mathcal{E}_{l}\right)$. There exist a Poncelet-Morley point associated to $M$.

Proof. The proof includes a construction programme of Poncelet-Morney.

Let $\left(\mathcal{E}_{l}\right)$ be an ellipse with focus $F$ and $F^{\prime} . M$ a point of $\left(\mathcal{E}_{l}\right)$. Let $\left(T_{l}\right)$ be the tangent at $M$ to $\left(T_{1}\right)$. Consider $A \neq M$ a point of $\left(T_{1}\right)$ such that $A F^{\prime}=F F^{\prime}$ and $M A$ is minimal.

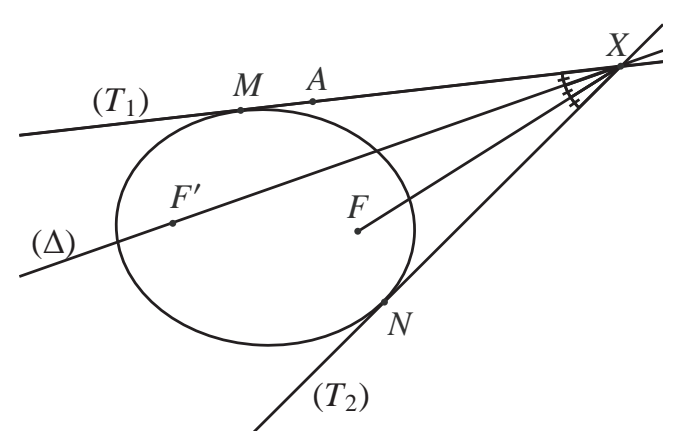

Therefore $F^{\prime}$ is on the median line of the segment $[A F]$. Let $X$ be the intersection of the bisector of angle $\widehat{F F^{\prime} A}$ and $\left(T_{1}\right)$. As $A F^{\prime}=F F^{\prime}$, the triangle $\triangle A F F^{\prime}$ is isosceles in $F^{\prime}$ thus $\left(F^{\prime} X\right)$ is the bisector of angle $\widehat{A F^{\prime} F}$ and median of the segment $[A F]$. Therefore the triangle $\triangle X A F$ is isosceles in $X$ and then $m e s \widehat{M X F^{\prime}}=m e s \widehat{A X F^{\prime}}=m e s \widehat{F^{\prime} X F}$. Let $\left(T_{2}\right)$ be another tangent at $N$ to $\left(\mathcal{E}_{l}\right)$ passing through $X$ and different from $\left(T_{1}\right)$. According to the second Poncelet theorem, $m e s \widehat{M X F^{\prime}}=m e s \widehat{F^{\prime} X F}=m e s \widehat{F X N}$. Then $X$ is the Poncelet-Morley point of $\left(\mathcal{E}_{l}\right)$ associated $M$.

\subsection{Number of Poncelet-Morley points associated with a given point of an ellipse}

In this section, $\left(\mathcal{E}_{l}\right)$ denotes a non degenerate ellipse of focus $F^{\prime}$ and $F ;\left(T_{1}\right)$ is a tangent at $M$ to $\left(\mathcal{E}_{l}\right)$ and $A$ is an arbitrary point of $\left(T_{1}\right)$ different to $M$. We denote by $[M A)$ the half-line of $\left(T_{1}\right)$ containing $A$.

Proposition 3. Let $\left(\mathcal{E}_{l}\right)$ be an ellipse of focus $F$ and $F^{\prime}$ and $M \in\left(\mathcal{E}_{l}\right)$. There exist at most two Poncelet-Morley points associated to $M$.

Proof. The proof also gives a construction programme.

The existence of a Poncelet-Morley point associated with $M$ is given by the above proposition. We remark that there is no Poncelet-Morley point if the bisector of the angle $\widehat{A F^{\prime} F}$ is parallel to $\left(T_{1}\right)$. 


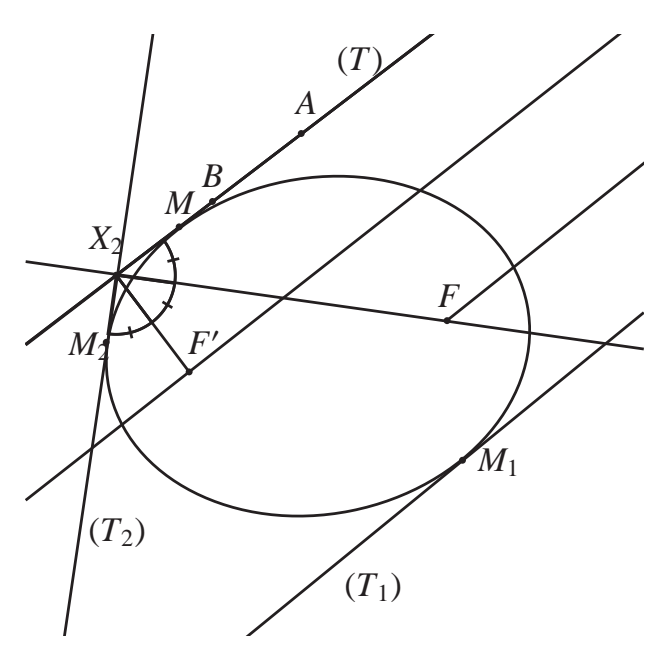

Let $B \in\left(T_{1}\right)-[M A)$ such that $F B=F^{\prime} F$. As in the proof of the above proposition, the bisector of $\widehat{B F F^{\prime}}$ intersect $\left(T_{1}\right)$ at a Poncelet-Morley point $X_{2}$.

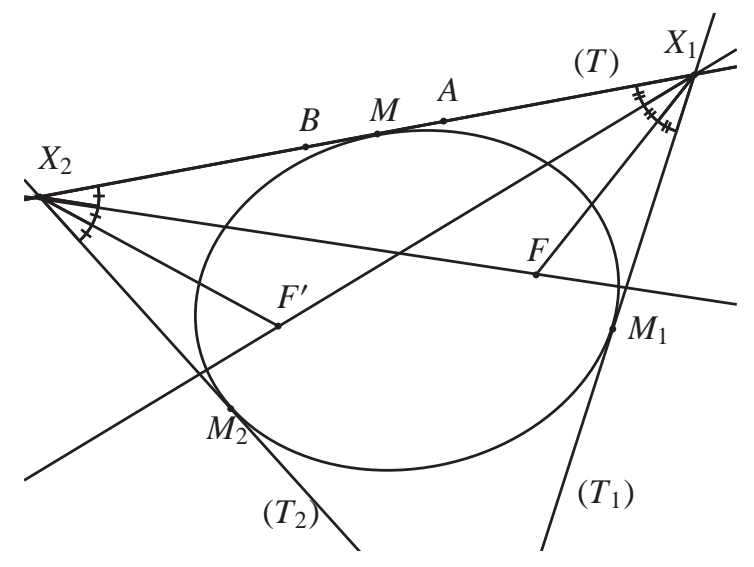

To place the point $B$ such as $F B=F^{\prime} F$.

To build the bisectrix of the angle $\widehat{B F F^{\prime}}$.

While reasoning like above, this bisectrix cuts $\left(T_{1}\right)$ in another point of Poncelet-Morney.

From where the existence (if possible) of the second point of Poncelet-Morney associated with $M$.

Suppose that there are three Poncelet-Morley points $\left(X_{1}, X_{2}, X_{3}\right)$ associated to $M$. Then two of them are on the same ray of $\left(T_{1}\right)$ with endpoint $M$. Suppose that $X_{1}$ and $X_{2}$ are two such points, then $\left(m e s \widehat{M X_{1} F^{\prime}}=m e s \widehat{F^{\prime} X_{1} F}\right.$ and mes $\widehat{M X_{2} F^{\prime}}=m e s \widehat{F^{\prime} X_{2} F}$.) Therefore $M^{\prime}$ is symmetric to $F$ relatively to $\left(F^{\prime} X_{1}\right)$ then $\left(F M^{\prime}\right) \perp\left(F^{\prime} X_{1}\right)$. If $M^{\prime \prime}$ is symmetric to $F$ relatively to $F^{\prime} X_{2}$ then $(F M ") \perp\left(F^{\prime} X_{2}\right)$.This implies that $F F^{\prime}=M^{\prime} F^{\prime}$ and $F F^{\prime}=M^{\prime \prime} F^{\prime}$ i.e; $F F^{\prime}=M^{\prime \prime} F^{\prime} \quad(1)$.

Since $X_{3}$ is a Poncelet-Morley point associated to $M, M^{\prime \prime} \in\left(T_{1}\right)$. But $M^{\prime}$ and $M$ "' are on $\left(T_{1}\right)$. According to (1), we conclude that $M^{\prime}=M^{\prime \prime}$. Therefore $X_{1}=X_{2}$. 


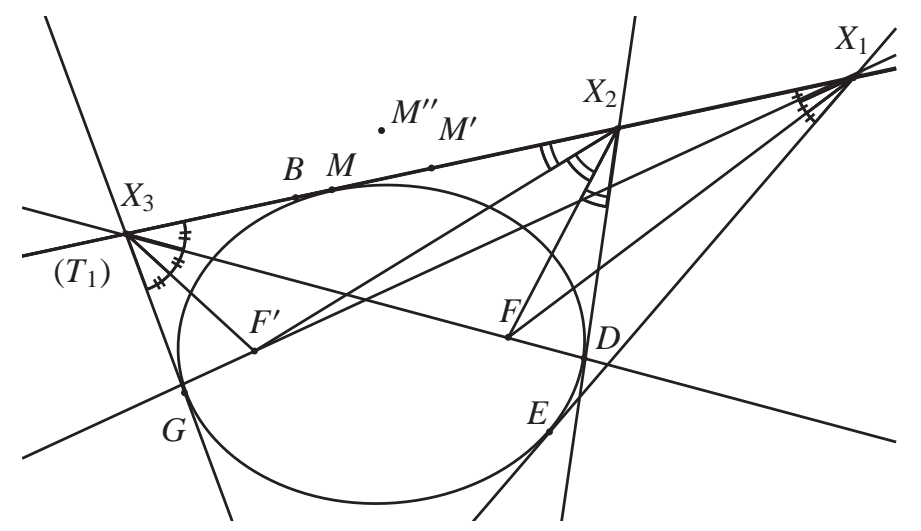

One can thus conclude that to each point of $\left(\mathcal{E}_{l}\right)$ we can associate at most two Poncelet-Morley points.

Theorem 4. If $X$ is a Poncelet-Morley point associated with $M \in\left(\mathcal{E}_{l}\right)$ and if $M^{\prime}$ and $X^{\prime}$ are respective images of $M$ and $X$ relatively to a symmetrical line of $\left(\mathcal{E}_{l}\right)$, then $X^{\prime}$ is a Poncelet-Morley point associated with $M^{\prime}$.

Proof. Since the orthogonal symmetry preserves the length and the measure of non oriented angle, the image of a tangent to $\left(\mathcal{E}_{l}\right)$ is another tangent and the image of the bisector of an angle is the bisector of the image of the angle.

Corollary 1. Given a non degenerated ellipse $\left(\mathcal{E}_{l}\right)$ with focus $F$ and $F^{\prime}$, there is an infinity Poncelet-Morley point associated to $\left(\mathcal{E}_{l}\right)$.

\section{Poncelet-morley Point and Angle Trisection Problem}

Angle trisection is a classic problem of compass and straightedge constructions of ancient Greek mathematics. It concerns the construction of an angle equal to one third of a given arbitrary angle, using only two tools: an unmarked straightedge, and a compass. The problem as stated is generally impossible to solve, as shown by Pierre-Laurent Wantzel in 1837; in (Pierre-LaurentWantzel, 1837). However, although there is no way to trisect an angle in general with just a compass and a straightedge, some special angles can be trisected; that is the case of a right angle. It is also possible to trisect an arbitrary angle by using tools other than straightedge and compass; that is the case of neusis construction; which is due to Archimedes.

Wantzel published his proof earlier in 1837 than Galois's, whose work was published in 1846. Therefore, his proof did not use the connection between field extensions and groups that is the subject of Galois theory itself.

According to Andrew M. Gleason in (Andrew M Gleason, 1988), the origin of this problem come from Gauss who discovered in (Carl Friedrich Gauss, 1966) how to construct a regular 17-gon using only ruler and compass. Gauss showed (Carl Friedrich Gauss, 1966) that regular polygons with 257 or 65537 sides can be constructed. He also stated without proof that no other regular polygons are constructible.

In what follows, we use the Poncelet-Morley point associated to any trisection problem of a given angle a cubic equation and use Galois theory to prove that the problem as stated is generally impossible to solve. If $\theta$ is a constructible angle, $3 \theta$ is also (it is the multiplication of the angles by three ). Conversely if $3 \theta$ is constructible is it always possible to construct $\theta$ ?

Our aim is to see if we can use the Poncelet-Morley point and give a solution to the problem of the trisection. In other words if $\widehat{A B C}$ is an angle is there any ellipse $\left(\mathcal{E}_{l}\right)$ such as $B$ is a Poncelet-Morley point associated with $A$ ? The answer to this question consists in the construction of the focus $F$ and $F^{\prime}$ of $\left(\mathcal{E}_{l}\right)$. Let $\widehat{A B C}$ be an acute angle such that $A B=B C$.

Let $D$ be a point of segment $[B C]$ and $(C)$ the circle of center $A$ passing through $B$.The parallel line $(\triangle)$ with $(A B)$ passing through $D$ intersect $(C)$ in two points. Indeed if $H$ is the orthogonal projection of $B$ on $(\Delta)$ then $B H<B D<B C=A B$ but $\widehat{A B C}$ is not a right angle. Consequently $d(A,(\Delta))=B H<A B=r$ the radius of the circle $(C)$. Then $(\Delta)$ meet $(C)$.

- Let $F^{\prime}$ be the intersection point of $(\Delta)$ and $(C)$ such that $D F^{\prime}$ is maximal and we note $\beta$ the angular measure $\widehat{A B F^{\prime}}$.

- When $D$ covers $[B C], \beta$ changes between 0 and $\frac{\alpha}{2}$. It can then take the value $\frac{\alpha}{3}$ 


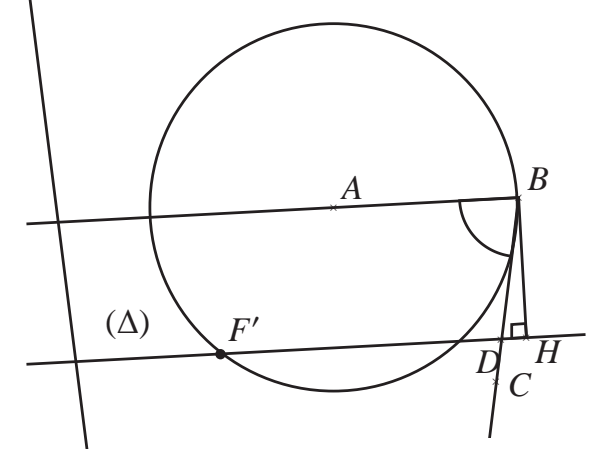

Therefore, the map $\theta:[B C] \longrightarrow\left[0, \frac{\alpha}{2}\right]$ which for each point $D \in[B C]$ associate the measure of $\widehat{A B F^{\prime}}$, is a bijection. We can then conclude that for all $\frac{\alpha}{3} \in\left[0, \frac{\alpha}{2}\right]$ there exist one and only one point $D \in[B C]$ such that $m e s \widehat{A B F^{\prime}}=\frac{\alpha}{3}$. This completes the algebraic proof of the existence of the trisection angle, but the trisection problem remains unsolved. That will be the case if we give the construction programme of the point $D$.

Since $D \in[B C]$ there exist $k \in] 0,1[$ such that $D$ is an image of $C$ by homothety with center $B$ and the ratio $k$. The trisection angle problem is then equivalent to the determination of ratio $k$.

$D$ is the image of $C$ by the homothety of center $B$ and ratio $k$;

Let $(\Delta)$ be the perpendicular line to $(A B)$ passing through $A$ and $\left(\Delta_{1}\right)$ the parallel line to $(A B)$ passing through $D$. We have $\left(\Delta_{1}\right) \cap(C)=F^{\prime}$ since $D F^{\prime}$ is maximum. Let $\left(\Delta_{2}\right)$ be the perpendicular line to $(B C)$ passing through $D$. We denote by $\left(\Delta_{1}^{\prime}\right)$ the image of $\left(\Delta_{1}\right)$ by the orthogonal symmetry relatively to $\left(\Delta_{2}\right)$. The image of the straight line $\left(A F^{\prime}\right)$ by orthogonal symmetry relatively to $(\Delta)$ intersects $\left(\Delta_{1}^{\prime}\right)$ at $F$. If $k$ is the solution of the problem, then $F$ and $F^{\prime}$ are focus of the ellipse tangent to $(A B)$ and $(B C)$ respectively at $A$ and $D$ such that angles $\widehat{A B F^{\prime}}, \widehat{F^{\prime} B F}$; and $\widehat{F B C}$ are congruent.

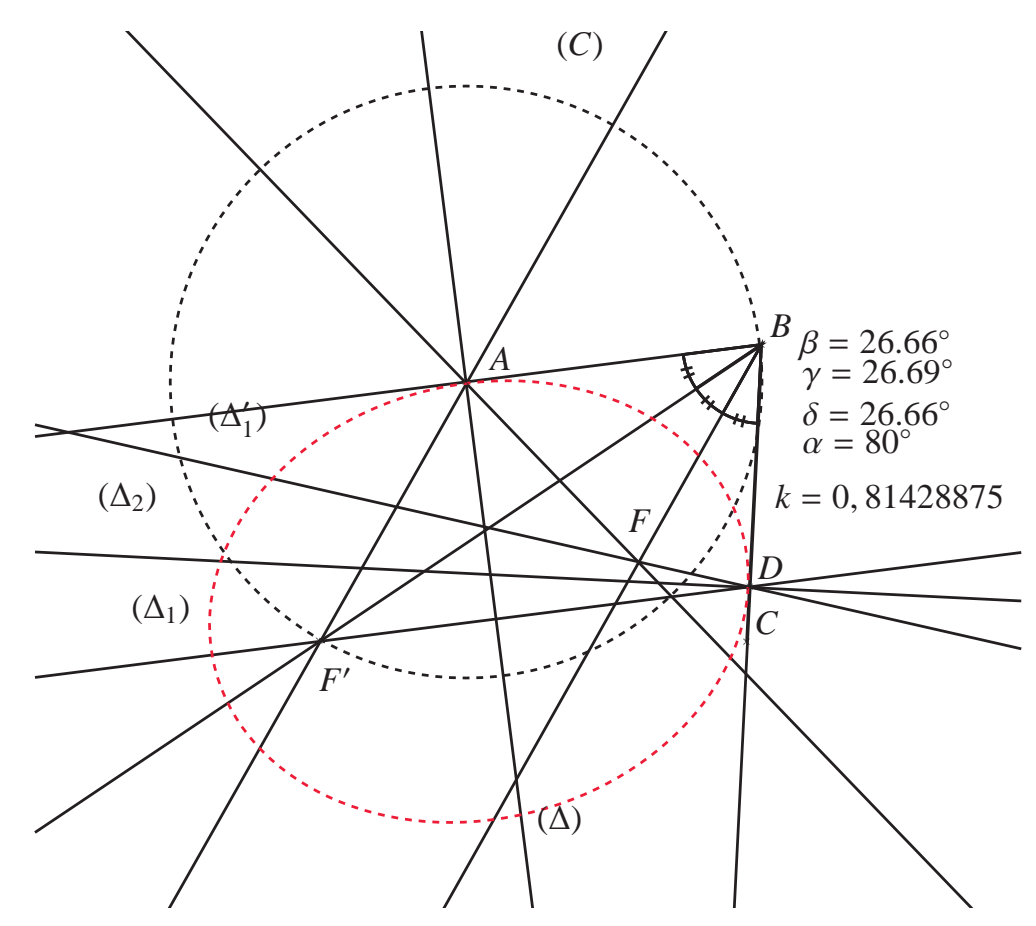

If $k$ is the solution of the problem then $B D=k A B$, since $A B=B C$. Therefore, in the isosceles triangle $\Delta_{A B F^{\prime}}$ one has: $B F^{\prime}=2 A B \cos \theta$. We deduce that $B D^{2}=B F^{\prime 2}+D F^{\prime 2}-2 B F^{\prime} \cdot D F^{\prime} \cos \theta$. This last equality implies that $k^{2} A B^{2}=$ 
$4 A B \cos ^{2} \theta\left(A B-D F^{\prime}\right)+D F^{2} \quad$ (1) Since

$$
\begin{aligned}
D F^{\prime 2} & =B D^{2}+B F^{2}-2 B F^{\prime} B D \cos 2 \theta \\
& =k^{2} A B^{2}+4 A B^{2} \cos ^{2} \theta-4 k A B^{2} \cos \theta \cos 2 \theta
\end{aligned}
$$

Then, by replacing (2) in (1) we obtain

$$
\begin{aligned}
& k^{2} A B^{2}=4 A B \cos ^{2} \theta\left(A B-\sqrt{k^{2} A B^{2}+4 A B^{2} \cos ^{2} \theta-4 k A B^{2} \cos \theta \cos 2 \theta}\right) \\
+ & k^{2} A B^{2}+4 A B^{2} \cos ^{2} \theta-4 k A B^{2} \cos \theta \cos 2 \theta \\
\text { i.e; } & 4 A B \cos ^{2} \theta\left(A B-A B \sqrt{k^{2}+4 \cos ^{2} \theta-4 k \cos \theta \cos 2 \theta}\right) \\
+ & 4 A B^{2} \cos ^{2} \theta-4 k A B^{2} \cos \theta \cos 2 \theta=0 \\
\text { i.e; } & 4 A B^{2} \cos \theta\left[\cos \theta\left(1-\sqrt{k^{2}-4 k \cos \theta \cos 2 \theta+4 \cos ^{2} \theta}\right)+\cos \theta-k \cos 2 \theta\right]=0 \\
\text { i.e; } & 2 \cos \theta-\cos \theta \sqrt{k^{2}-4 k \cos \theta \cos 2 \theta+4 \cos ^{2} \theta}-k \cos 2 \theta=0 \\
\text { i.e; } & 2 \cos \theta-k \cos 2 \theta=\cos \theta \sqrt{k^{2}-4 k \cos \theta \cos ^{2} \theta+4 \cos ^{2} \theta} \\
\text { i.e; } & 4 \cos { }^{2} \theta-4 k \cos \theta \cos 2 \theta+k^{2} \cos ^{2} 2 \theta=\cos ^{2} \theta\left(k^{2}-4 k \cos \theta \cos 2 \theta+4 \cos ^{2} \theta\right) \\
\text { i.e; } & 4 \cos ^{2} \theta-4 k \cos \theta \cos 2 \theta+k^{2} \cos ^{2} 2 \theta=k^{2} \cos ^{2} \theta-4 \cos ^{3} \theta \cos 2 \theta+4 \cos ^{4} \theta \\
\text { i.e; } & k^{2}\left(\cos ^{2} 2 \theta-\cos 2 \theta\right)-4 k\left(\cos \theta \cos ^{2} 2 \theta-\cos ^{3} \theta \cos 2 \theta\right)+4 \cos ^{2} \theta\left(1-\cos ^{2} \theta\right)=0
\end{aligned}
$$

Let us denote $a(\theta)=3 \cos ^{2} \theta-1, b(\theta)=-4 \cos 2 \theta\left(\cos \theta-\cos ^{3} \theta\right)$ and $c(\theta)=4 \cos ^{2} \theta\left(1-\cos ^{2} \theta\right)$. The (E) becomes

$$
a(\theta) k^{2}+b(\theta) k+c(\theta)=0
$$

The solutions of this equation depend on $\cos \theta$. The trisection angle problem will be solved if we can construct the real solutions of equation (E). But according to the Galois theory there are many real numbers which cannot be constructed using only a compass and an unmarked straightedge. This allows us to conclude that The problem as stated is generally impossible to solve.

\section{Acknowledgement}

We will like to thank Mister Shuntah Roland Yotcha and Delphine Tarsiwang who carry out the necessary English translation.

\section{References}

Andrew M. Gleason. (1988). Angle trisection, the heptagon, and the triskaidecagon. Amer. Math. Monthly, 95(3), 185-194. https://doi.org/10.2307/2323624

Carl Friedrich Gauss. (1966). Disquisitiones arithmeticae, 157. Yale University Press, Msc200: 51M04,

Ian Nicholas Stewart. (2015). Galois theory. CRC Press.

Jean Fresnel. (1996). Méthodes modernes en géométrie. Hermann.

Jim Loy. (2003). Trisection of an angle.

Pierre-LaurentWantzel. (1837). Recherches sur les moyens de reconna?tre si un problème de géométrie peut se résoudre avecla règle et le compas. Journal de Mathématiques pures et appliquées, 2(1), 366-372.

Robert C Yates. (1942). The trisection problem. National Mathematics Magazine, 16(4), 171-182. https://doi.org/10.2307/3028266

\section{Copyrights}

Copyright for this article is retained by the author(s), with first publication rights granted to the journal.

This is an open-access article distributed under the terms and conditions of the Creative Commons Attribution license (http://creativecommons.org/licenses/by/4.0/). 